OPEN ACCESS

Edited by:

Elena Lazzeri,

University of Florence, Italy

Reviewed by:

Hideyuki Saito,

Kumamoto University Hospital, Japan

Letizia De Chiara,

University of Florence, Italy

*Correspondence:

Daniel A. Heller

hellerd@mskcc.org

Edgar A. Jaimes

jaimese@mskcc.org

Specialty section:

This article was submitted to

Renal Pharmacology,

a section of the journal

Frontiers in Pharmacology

Received: 07 October 2021

Accepted: 30 November 2021

Published: 03 January 2022

Citation:

Williams RM, Shah J, Mercer E, Tian HS, Thompson V, Cheung JM, Dorso M, Kubala JM, Gudas LJ,

de Stanchina E, Jaimes EA and

Heller DA (2022) Kidney-Targeted

Redox Scavenger Therapy Prevents

Cisplatin-Induced Acute Kidney Injury.

Front. Pharmacol. 12:790913.

doi: 10.3389/fphar.2021.790913

\section{Kidney-Targeted Redox Scavenger Therapy Prevents Cisplatin-Induced Acute Kidney Injury}

Ryan M. Williams ${ }^{1,2}$, Janki Shah ${ }^{2}$, Elizabeth Mercer ${ }^{2}$, Helen S. Tian ${ }^{2}$, Vanessa Thompson ${ }^{2}$, Justin M. Cheung ${ }^{2}$, Madeline Dorso ${ }^{2,3}$, Jaclyn M. Kubala ${ }^{2,3}$, Lorraine J. Gudas ${ }^{4}$, Elisa de Stanchina ${ }^{2}$, Edgar A. Jaimes ${ }^{2,3 *}$ and Daniel A. Heller ${ }^{2,3 *}$

${ }^{1}$ The City College of New York Department of Biomedical Engineering, New York, NY, United States, ${ }^{2}$ Memorial Sloan Kettering Cancer Center, New York, NY, United States, ${ }^{3}$ Weill Cornell Medical College, New York, NY, United States, ${ }^{4}$ Department of Pharmacology, Weill Cornell Medical College, New York, NY, United States

Cisplatin-induced acute kidney injury (Cl-AKI) is a significant co-morbidity of chemotherapeutic regimens. While this condition is associated with substantially lower survival and increased economic burden, there is no pharmacological agent to effectively treat $\mathrm{Cl}-\mathrm{AKI}$. The disease is hallmarked by acute tubular necrosis of the proximal tubular epithelial cells primarily due to increased oxidative stress. We investigated a drug delivery strategy to improve the pharmacokinetics of an approved therapy that does not normally demonstrate appreciable efficacy in $\mathrm{Cl}-\mathrm{AKI}$, as a preventive intervention. In prior work, we developed a kidney-selective mesoscale nanoparticle (MNP) that targets the renal proximal tubular epithelium. Here, we found that the nanoparticles target the kidneys in a mouse model of $\mathrm{Cl}-\mathrm{AKI}$ with significant damage. We evaluated MNPs loaded with the reactive oxygen species scavenger edaravone, currently used to treat stroke and ALS. We found a marked and significant therapeutic benefit with edaravone-loaded MNPs, including improved renal function, which we demonstrated was likely due to a decrease in tubular epithelial cell damage and death imparted by the specific delivery of edaravone. The results suggest that renal-selective edaravone delivery holds potential for the prevention of acute kidney injury among patients undergoing cisplatin-based chemotherapy.

Keywords: nanomedicine, pharmacology, acute kidney injury, drug repurposing, cisplatin, redox scavenger

\section{INTRODUCTION}

Acute kidney injury (AKI) is a common clinical condition associated with significant morbidity and mortality regardless of etiology or setting. AKI affects millions of individual patients and has a large socioeconomic impact, including longer hospital stay and higher costs. In the United States alone, it is estimated that the annual costs related to AKI are up to $\$ 24$ billion (Silver and Chertow, 2017). The incidence of AKI is increasing at a rapid pace (Xue et al., 2006; Goldberg and Dennen, 2008), which is attributable to several factors including shifts in demographics, severity of underlying diseases, and expansion of invasive and complex medical procedures (Hoste and Schurgers, 2008; Zappitelli, 2008). AKI can result from a variety of insults including volume depletion, septicemia, hypotension, and commonly used drugs including antibiotics and chemotherapeutic agents.

Cisplatin is a widely used chemotherapy in the treatment of a variety of cancers including ovarian, head and neck, bladder, testicular, and lung, among others (Belani, 2004; Latcha et al., 2016). A 
significant side effect of cisplatin therapy is AKI which is seen in approximately 33\% of patients (Ozkok and Edelstein, 2014; Latcha et al., 2016). Importantly, the development of AKI in these patients can result in the interruption of chemotherapy or a change to less effective chemotherapies (Ozkok and Edelstein, 2014; Latcha et al., 2016). There is therefore a critical need to develop novel strategies to prevent or treat AKI induced by cisplatin, which would also potentially have a direct impact on the oncologic outcomes of these patients whose treatment otherwise cannot be completed, or other less-effective chemotherapeutic agents must be used (Al-Mamgani et al., 2017).

There has been significant overall progress in our understanding of the epidemiology, pathophysiology and outcomes of AKI. Despite such substantial advances, almost no meaningful progress has been made in the treatment of AKI and there are no effective pharmacologic approaches currently approved for the treatment of AKI (Bellomo et al., 2012). Hence, clinicians rely on conservative measures and renal replacement therapy if indicated for the management of AKI (Greenberg and Cheung, 2005). Numerous strategies have been proposed to prevent, ameliorate, or treat AKI. These include inhibition of inflammatory mediators, enhancement of renal perfusion by blocking vasoconstrictor mechanisms and/or enhancing vasodilation, attenuation of leukocyte infiltration, inhibition of the coagulation cascade, inhibition of reactive oxygen species (ROS), and administration of growth factors to accelerate renal recovery (Zarjou et al., 2012; Humphreys et al., 2015). Most of these attempts have shown moderate success in attenuating AKI in animal models but have subsequently failed in clinical trials. The reasons for these failures are multifactorial but often include poor delivery of therapeutic agents to the proximal tubules, systemic toxicity at doses required to have a therapeutic effect, concomitant co-morbid conditions, suboptimal clinical trial design, and heterogeneity in cause and timing of AKI, among others (Awdishu and Joy, 2016).

We previously developed a mesoscale nanoparticle (MNP) (Williams et al., 2015; Williams et al., 2018), a drug carrier that localizes therapeutic and imaging payloads to the kidneys. The nanoparticles specifically target the renal cortex tubular epithelium, localizing in both proximal tubular and distal tubular epithelial cells. In our prior work, we investigated the pharmacokinetics and mechanism of renal localization of the particles themselves via in vivo and ex vivo imaging experiments (Williams et al., 2015; Williams et al., 2018). We found that renal targeting of MNPs depended on their size (350-400 nm) and surface chemistry (polyethylene glycol-coated surface) (Williams et al., 2015; Williams et al., 2018), whereas other sizes or surface modifications exhibited primarily liver accumulation. These studies utilized MNPs loaded with a fluorescent dye and found that the particles are likely too large for glomerular filtration and reabsorption through the luminal membrane of tubular epithelial cells. Instead, they suggested that particles reach the tubular epithelial cells via transcytosis across the peritubular capillaries and were taken up by tubular epithelial cells at their basolateral membrane, similar to previously-studied mechanisms (Stamatiades et al., 2016). As these particles have no specific targeting moiety for the peritubular endothelium or tubular epithelium, we hypothesized that this uptake was primarily driven by a decrease in fluid flow in the peritubular capillary combined with a large increase in absorptive pressure of peritubular capillaries (Brenner et al., 1971; Aird, 2007). Further, the particles were found to be biodegradable, releasing cargoes over days to weeks, and they exhibited minimal toxicity (Williams et al., 2018). The MNP drug carrier platform has been investigated in ischemia-reperfusion injury, using experimental drug payloads including peptides and oligodinucleotides (Han et al., 2020a; Han et al., 2020b).

No clinical methods currently exist to target the majority of a therapeutic specifically to the site of CI-AKI (Jo et al., 2007; Williams et al., 2016). Most attempted therapeutic strategies for CI-AKI have been hindered by side effects and/or poor drug accumulation at the site of injury (Walz et al., 2010; Di Lorenzo et al., 2011; Gewin et al., 2012). Reactive oxygen species (ROS) scavenger therapy, for instance, has been investigated in the treatment of CI-AKI, as oxidative stress is a predominant mechanism of injury in cisplatin-induced AKI (Ozkok and Edelstein, 2014; Golombek et al., 2018), but successful attempts required high doses to effect efficacious responses in rodents (Iguchi et al., 2004), suggesting that human trials would result in dose-limiting toxicities. If strategies existed to change drug pharmacokinetics to localize therapies to the proximal renal tubules, therapeutic efficacy would likely be significantly improved (Zarjou et al., 2012). Additionally, it is hypothesized that therapeutic intervention proximal to the time of cisplatin infusion would be most efficacious in the treatment of CI-AKI, due to the rapid onset of tubular necrosis after cisplatin dosing and the poor timing of AKI diagnosis and therapy initiation (Walz et al., 2010; Di Lorenzo et al., 2011; Gewin et al., 2012). The pathophysiology of CI-AKI therefore warrants preventive therapy, if the intervention were to exhibit minimal risk of toxicities.

Here, we investigated the potential for preventive therapy of CI-AKI via kidney-targeted delivery of free radical scavenger treatment. The free radical scavenger edaravone, approved in the United States for ALS and in Japan for ischemic stroke and ALS (Lapchak, 2010; Voelker, 2017), was encapsulated in the MNP drug carrier. We found that edaravone-loaded MNPs targeted the kidneys in a CI-AKI mouse model, improved the pharmacokinetics of edaravone, and imparted substantial protection against CI-AKI as measured by renal function, histology, and oxidative stress levels.

\section{MATERIALS AND METHODS}

\section{MNP Formulation}

Mesoscale nanoparticles were formulated from a di-block PLGAPEG copolymer similarly to described in our prior work (Williams et al., 2015; Williams et al., 2018). PLGA-PEG was synthesized via conjugation of carboxylic acid-terminated poly(lactic-co-glycolic acid) (PLGA) and heterobifunctional polyethylene glycol $\left(\mathrm{NH}_{2}\right.$-PEG-COOH). PLGA (50:50; $\mathrm{MW}$ $38-54 \mathrm{kDa} ; 5 \mathrm{~g}$; Aldrich, St. Louis, MO) was dissolved in $10 \mathrm{ml}$ methylene chloride and mixed with $\mathrm{N}$-hydroxysuccinimide 
TABLE 1 | Physical characteristics of MNPs used in all studies.

\begin{tabular}{|c|c|c|c|c|c|}
\hline Formulation & $\begin{array}{l}\text { Hydrodynamic diameter } \\
\text { (nm) }\end{array}$ & PDI & $\begin{array}{c}\% \text { within } \\
164-531 \mathrm{~nm}\end{array}$ & 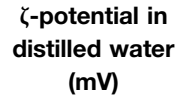 & $\begin{array}{c}\zeta \text {-potential in } \\
100 \% \text { serum } \\
\text { (mV) }\end{array}$ \\
\hline Eda-MNPs & $374.0(12.2)$ & $0.337(0.032)$ & 85 & $-22.8(0.55)$ & $-18.2(0.51)$ \\
\hline Ctrl-MNPs & $332.0(7.56)$ & $0.302(0.040)$ & 74 & $-24.7(0.61)$ & $-13.2(0.70)$ \\
\hline
\end{tabular}

(NHS; $135 \mathrm{mg} ;$ Aldrich) and 1-ethyl-3-(3-(dimethylamino) propyl)-carbodiimide (EDC; $230 \mathrm{mg}$; Aldrich) overnight. Activated PLGA-NHS was precipitated with cold ethyl ether and washed $3 \times$ with cold 50:50 ethyl ether:methanol before drying under vacuum. Dried PLGA-NHS ( $1 \mathrm{~g}$ ) was dissolved in chloroform with heterobifunctional PEG (250 mg; Nanocs, New York, NY) with $37 \mu \mathrm{l}$, N-diisopropylethylamine and mixed overnight. PLGA-PEG was precipitated with cold methanol and washed $3 \times$ with the same before drying under vacuum. Conjugation was confirmed via ${ }^{1} \mathrm{H}$ NMR as previously described (Cheng et al., 2007).

MNPs were formulated via nanoprecipitation. PLGA-PEG $(100 \mathrm{mg})$ was dissolved in acetonitrile $(2 \mathrm{ml})$ with either edaravone (100 mg; Santa Cruz Biotechnology, Dallas, TX) for Eda-MNPs, or with no co-precipitate molecule for Ctrl-MNPs. This was added dropwise $(100 \mu \mathrm{l} / \mathrm{min})$ to purified water $(4 \mathrm{ml})$ with Pluronic F-68 (100 $\mu$ l; Life Technologies, Carlsbad, CA). Each batch of particles was stirred for $2 \mathrm{~h}$ prior to centrifugation at $7356 \mathrm{RCF}$ for $15 \mathrm{~min}$ prior to washing with purified water and centrifuging again. The recovered MNPs were suspended in a $2 \%$ sucrose solution and lyophilized until a fluffy powder was obtained.

\section{MNP Characterization}

MNP batches were characterized to determine their size and charge. Size and polydispersity index (PDI) were measured via dynamic light scattering (DLS; Malvern, Worcestershire, United Kingdom) in $1 \mathrm{x}$ phosphate buffered saline (PBS) at $10 \mathrm{mg} / \mathrm{ml}$. Surface charge was measured via electrophoretic light scattering (ELS; Malvern) in purified water at $1 \mathrm{mg} / \mathrm{ml}$. The effect of serum on surface charge was determined by incubating each batch at $1 \mathrm{mg} / \mathrm{ml}$ in $100 \%$ fetal bovine serum (FBS) at room temperature for $30 \mathrm{~min}$, centrifuging at $7356 \mathrm{RCF}$ for $15 \mathrm{~min}$, and resuspending the particle pellet in purified water for ELS. For each size and charge measurements, particle batches were measured three times and the mean \pm standard deviation as reported.

Cargo loading and release were also characterized for EdaMNPs. Cargo loading was determined by dissolving particles in acetonitrile, centrifuging at $31,000 \mathrm{RCF}$ for $15 \mathrm{~min}$ to remove polymer, and performing UV-VIS spectrophotometry (Jasco, Easton, MD) on the supernatant. Cargo release was determined by suspending particles at $10 \mathrm{mg} / \mathrm{ml} \mathrm{PBS}$ and incubating for $72 \mathrm{~h}$ at $37^{\circ} \mathrm{C}$ or room temperature. At $2,4,6,24$, and $48 \mathrm{~h}$, as well as the final $72 \mathrm{~h}$ timepoint, particles were centrifuged and the supernatant measured via UV-VIS spectrophotometry.

\section{Cisplatin-Induced AKI Mouse Model}

To next investigate the therapeutic efficacy of Eda-MNPs, we recapitulated a model of cisplatin-induced acute kidney injury as previously described (Kim et al., 2012). Briefly, male 8-12-weeks C57BL/6 mice (Charles River, Troy, NY) were deprived of food and water for $18 \mathrm{~h}$ prior to induction. We used male mice only as female mice are more resistant to renal injury (Müller et al., 2002; Wei et al., 2005; Yang et al., 2010). Cisplatin (Sigma) was prepared for injection by dissolving at $1 \mathrm{mg} / \mathrm{ml}$ in sterile saline followed by a 30 -min incubation in a $37^{\circ} \mathrm{C}$ water bath to ensure dissolution while protecting from light. Mice were then injected intraperitoneally with $25 \mathrm{mg} / \mathrm{kg}$, at which time food and water were returned. Mice were sacrificed $72 \mathrm{~h}$ following cisplatin injection following a terminal retroorbital bleed. In addition to serum collection, kidneys were harvested and prepared for further study. Healthy control groups either underwent fasting and dehydration with no cisplatin injection or received food and water ad libitum.

\section{Therapeutic Effect of Eda-MNPs in CI-AKI}

We then sought to determine the therapeutic efficacy of EdaMNPs in mice with CI-AKI. We investigated six total mouse groups $(N=5-10)$, including negative controls, positive controls, two unencapsulated drug groups, empty MNPs, and Eda-MNPs as the investigative group: 1) Healthy control mice; 2) CI-AKI; 3) $\mathrm{CI}-\mathrm{AKI}+50 \mathrm{mg} / \mathrm{kg}$ Eda-MNPs (intravenous in PBS); 4) CI-AKI $+0.2 \mathrm{mg} / \mathrm{kg}$ free edaravone (intravenous in PBS; matched dose); 5) $\mathrm{CI}-\mathrm{AKI}+30 \mathrm{mg} / \mathrm{kg}$ free edaravone (intravenous in PBS; high dose); and 6) CI-AKI + $50 \mathrm{mg} / \mathrm{kg}$ Ctrl-MNPs (intravenous in PBS). All treatments were performed $24 \mathrm{~h}$ after cisplatin injection prior to sacrifice at $72 \mathrm{~h}$ and organ formalin fixing and staining as above. Mice were weighed at the time of food and water removal, at cisplatin administration, and every $24 \mathrm{~h}$ afterwards until sacrifice.

We also performed a therapeutic efficacy study in mice with CI-AKI that were not fasted and dehydrated to further confirm the potential clinical utility of this therapeutic tool. In this experiment, cisplatin was injected into mice as above, though we only investigated negative control, positive control, and investigational therapeutic groups $(N=3-5)$ : 1) Healthy control mice; 2) CI-AKI; and 3) CI-AKI + $50 \mathrm{mg} / \mathrm{kg} \mathrm{Eda-}$ MNPs. MNPs were administered via the tail vein $24 \mathrm{~h}$ after CI-AKI induction. Serum was collected for further study $72 \mathrm{~h}$ after cisplatin administration at the time of sacrifice.

\section{Mass Spectrometry Analysis of Blood and Renal Tissue}

To determine the systemic and local concentrations of edaravone facilitated by MNP delivery, we performed liquid chromatography-mass spectrometry (LC-MS). Four healthy 

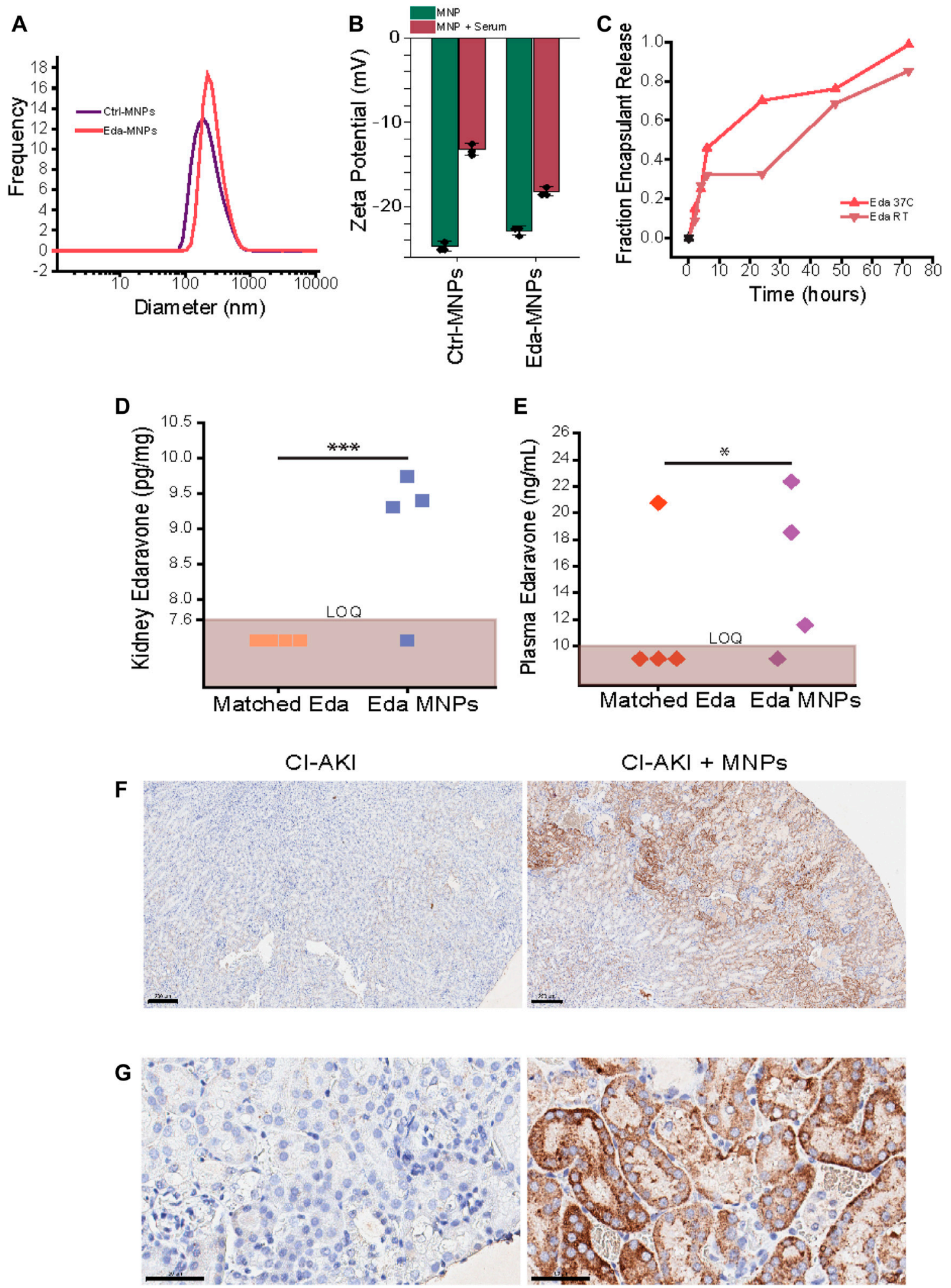

FIGURE 1 | Encapsulation and kidney-selective delivery of edaravone by polymeric mesoscale nanoparticles. (A) Hydrodynamic diameter distribution of Ctrl-MNPs and Eda-MNPs used in all studies. (B) $\zeta$-potential of MNPs used in all studies in water versus after incubation with fetal bovine serum. (C) Percent release edaravone at room temperature (RT) or $37^{\circ} \mathrm{C}$ over time. (D) LC-MS quantification of edaravone levels as a function of renal mass. LOQ represents approximately 7.6 pg/mg. Each data point represents a single mouse, and data points in the brown zone signify they were below LOQ. A chi-squared test was performed to determine the significance between the number of values above and below LOQ threshold; $p={ }^{\star \star \star}=5.32 \mathrm{E}^{-4}$. (E) LC-MS quantification of edaravone levels as a function of total recovered plasma 
FIGURE 1 | volume. LOQ represents approximately $10 \mathrm{ng} / \mathrm{ml}$. Each data point represents a single mouse, and data points in the brown zone signify they were below LOQ. A chi-squared test was performed to determine the significance between the number of values above and below $L O Q$ threshold; $p={ }^{*}=0.021$. (F) Representative immunohistochemistry (at $\times 5$ magnification) with anti-PEG antibody staining for MNP accumulation in renal tissue observing part of cortex and medulla. DAB detection (brown) and counterstained with haematoxylin (blue). Scale bar $=200 \mu \mathrm{m}$. (G) $\times 40$-magnification image of primarily cortex from panel (F). Scale bar $=50 \mu \mathrm{m}$.

TABLE 2 | Individual mouse data from therapeutic efficacy studies (note: a negative weight loss percentage represents a weight gain).

\begin{tabular}{|c|c|c|c|c|c|c|c|c|c|c|c|c|}
\hline Treatment & $\begin{array}{c}\text { Age at } \\
\text { euthanasia } \\
\text { (Days) }\end{array}$ & $\begin{array}{l}\text { Pre- } \\
\text { dehydration } \\
\text { weight } \\
\text { (g) }\end{array}$ & $\begin{array}{l}\text { Pre- } \\
\text { cisplatin } \\
\text { weight } \\
\text { (g) }\end{array}$ & $\begin{array}{c}\text { Day } 1 \\
\text { weight } \\
\text { (g) }\end{array}$ & $\begin{array}{c}\text { Day } 2 \\
\text { weight } \\
\text { (g) }\end{array}$ & $\begin{array}{c}\text { Day } 3 \\
\text { weight } \\
\text { (g) }\end{array}$ & $\begin{array}{l}\text { Pre- } \\
\text { cisplatin } \\
\text { weight } \\
\text { loss } \\
(\%)\end{array}$ & $\begin{array}{c}\text { Day } 1 \\
\text { weight } \\
\text { loss } \\
(\%)\end{array}$ & $\begin{array}{c}\text { Day } 2 \\
\text { weight } \\
\text { loss } \\
(\%)\end{array}$ & $\begin{array}{c}\text { Day } 3 \\
\text { weight } \\
\text { loss } \\
(\%)\end{array}$ & $\begin{array}{c}\text { Serum } \\
\text { creatinine } \\
\text { (mg/dl) }\end{array}$ & $\begin{array}{c}\text { Blood } \\
\text { urea } \\
\text { nitrogen } \\
\text { (mg/dl) }\end{array}$ \\
\hline Healthy & 94 & & & & & & & & & & 0.16 & 32 \\
\hline Healthy & 94 & & & & & & & & & & NA & 21 \\
\hline Healthy & 94 & & & & & & & & & & 0.12 & 23 \\
\hline Healthy & 94 & & & & & & & & & & 0.16 & 22 \\
\hline Healthy & 94 & & & & & & & & & & 0.15 & 21 \\
\hline Healthy & 103 & 25 & 24 & 30 & 28 & 28 & 4.00 & -20.00 & -12.00 & -12.00 & 0.18 & 26 \\
\hline Healthy & 103 & 27 & 24 & 27 & 26 & 27 & 11.11 & 0.00 & 3.70 & 0.00 & 0.15 & 29 \\
\hline Healthy & 103 & 28 & 24 & 28 & 28 & 28 & 14.29 & 0.00 & 0.00 & 0.00 & 0.18 & 28 \\
\hline Healthy & 103 & 29 & 26 & 30 & 31 & 30 & 10.34 & -3.45 & -6.90 & -3.45 & 0.19 & 29 \\
\hline Healthy & 103 & 27 & 25 & 26 & 26 & 26 & 7.41 & 3.70 & 3.70 & 3.70 & 0.20 & 28 \\
\hline Cisplatin & 72 & 27 & 23 & 23 & 21 & 20 & 14.81 & 14.81 & 22.22 & 25.93 & 1.52 & 241 \\
\hline Cisplatin & 72 & 28 & 24 & 24 & 23 & 21 & 14.29 & 14.29 & 17.86 & 25.00 & 0.22 & 111 \\
\hline Cisplatin & 72 & 27 & 23 & 22 & 21 & 20 & 14.81 & 18.52 & 22.22 & 25.93 & 0.95 & 249 \\
\hline Cisplatin & 72 & 26 & 21 & 20 & 19 & 18 & 19.23 & 23.08 & 26.92 & 30.77 & 0.35 & 232 \\
\hline Cisplatin & 72 & 28 & 26 & 26 & 24 & 23 & 7.14 & 7.14 & 14.29 & 17.86 & 1.1 & 243 \\
\hline Cisplatin & 86 & 30 & 26 & 26 & 24 & 23 & 13.33 & 13.33 & 20.00 & 23.33 & 0.52 & 198 \\
\hline Cisplatin & 86 & 27 & 23 & 23 & 20 & 20 & 14.81 & 14.81 & 25.93 & 25.93 & 0.35 & 91 \\
\hline Cisplatin & 79 & 28 & 25 & 24 & 23 & 22 & 10.71 & 14.29 & 17.86 & 21.43 & 0.45 & 115 \\
\hline Cisplatin & 79 & 27 & 26 & 26 & 24 & 21 & 3.70 & 3.70 & 11.11 & 22.22 & 0.08 & 85 \\
\hline $\begin{array}{l}\text { Cis + Eda } \\
\text { MNPs }\end{array}$ & 72 & 27 & 23 & 23 & 23 & 20 & 14.81 & 14.81 & 14.81 & 25.93 & 0.09 & 30 \\
\hline $\begin{array}{l}\text { Cis + Eda } \\
\text { MNPs }\end{array}$ & 72 & 29 & 25 & 26 & 25 & 23 & 13.79 & 10.34 & 13.79 & 20.69 & 0.08 & 44 \\
\hline $\begin{array}{l}\text { Cis + Eda } \\
\text { MNPs }\end{array}$ & 72 & 30 & 25 & 24 & 24 & 22 & 16.67 & 20.00 & 20.00 & 26.67 & 0.08 & 51 \\
\hline $\begin{array}{l}\text { Cis + Eda } \\
\text { MNPs }\end{array}$ & 72 & 27 & 23 & 24 & 23 & 22 & 14.81 & 11.11 & 14.81 & 18.52 & 0.13 & 38 \\
\hline $\begin{array}{l}\text { Cis + Eda } \\
\text { MNPs }\end{array}$ & 72 & 29 & 26 & 27 & 25 & 22 & 10.34 & 6.90 & 13.79 & 24.14 & 0.07 & 32 \\
\hline $\begin{array}{l}\text { Cis + Eda } \\
\text { MNPs }\end{array}$ & 79 & 27 & 24 & 22 & 23 & 22 & 11.11 & 18.52 & 14.81 & 18.52 & 0.34 & 126 \\
\hline $\begin{array}{l}\text { Cis + Eda } \\
\text { MNPs }\end{array}$ & 79 & 28 & 25 & 21 & 23 & 21 & 10.71 & 25.00 & 17.86 & 25.00 & 0.03 & 55 \\
\hline $\begin{array}{l}\text { Cis + Eda } \\
\text { MNPs }\end{array}$ & 79 & 27 & 25 & 24 & 23 & 21 & 7.41 & 11.11 & 14.81 & 22.22 & 0.13 & 92 \\
\hline $\begin{array}{l}\text { Cis + Eda } \\
\text { MNPs }\end{array}$ & 79 & 27 & 24 & 23 & 23 & 21 & 11.11 & 14.81 & 14.81 & 22.22 & 0.07 & 110 \\
\hline $\begin{array}{l}\text { Cis + Eda } \\
\text { MNPs }\end{array}$ & 79 & 27 & 24 & 24 & 22 & 20 & 11.11 & 11.11 & 18.52 & 25.93 & 0.04 & 50 \\
\hline $\begin{array}{l}\text { Cis + High } \\
\text { Dose Eda }\end{array}$ & 72 & 30 & 26 & 26 & 25 & 24 & 13.33 & 13.33 & 16.67 & 20.00 & 0.13 & 59 \\
\hline $\begin{array}{l}\text { Cis + High } \\
\text { Dose Eda }\end{array}$ & 72 & 27 & 23 & 23 & 23 & 21 & 14.81 & 14.81 & 14.81 & 22.22 & 0.09 & 45 \\
\hline $\begin{array}{l}\text { Cis + High } \\
\text { Dose Eda }\end{array}$ & 72 & 25 & 23 & 23 & 25 & 22 & 8.00 & 8.00 & 0.00 & 12.00 & 0.18 & 36 \\
\hline $\begin{array}{l}\text { Cis + High } \\
\text { Dose Eda }\end{array}$ & 72 & 28 & 24 & 25 & 23 & 21 & 14.29 & 10.71 & 17.86 & 25.00 & 0.09 & 61 \\
\hline $\begin{array}{l}\text { Cis + High } \\
\text { Dose Eda }\end{array}$ & 72 & 29 & 26 & 24 & 25 & 23 & 10.34 & 17.24 & 13.79 & 20.69 & 0.13 & 59 \\
\hline $\begin{array}{l}\text { Cis + High } \\
\text { Dose Eda }\end{array}$ & 86 & 31 & 26 & 26 & 22 & 22 & 16.13 & 16.13 & 29.03 & 29.03 & 1.87 & 286 \\
\hline
\end{tabular}


TABLE 2 | (Continued) Individual mouse data from therapeutic efficacy studies (note: a negative weight loss percentage represents a weight gain).

\begin{tabular}{|c|c|c|c|c|c|c|c|c|c|c|c|c|}
\hline Treatment & $\begin{array}{c}\text { Age at } \\
\text { euthanasia } \\
\text { (Days) }\end{array}$ & $\begin{array}{l}\text { Pre- } \\
\text { dehydration } \\
\text { weight } \\
\text { (g) }\end{array}$ & $\begin{array}{l}\text { Pre- } \\
\text { cisplatin } \\
\text { weight } \\
\text { (g) }\end{array}$ & $\begin{array}{c}\text { Day } 1 \\
\text { weight } \\
\text { (g) }\end{array}$ & $\begin{array}{c}\text { Day } 2 \\
\text { weight } \\
\text { (g) }\end{array}$ & $\begin{array}{c}\text { Day } 3 \\
\text { weight } \\
\text { (g) }\end{array}$ & $\begin{array}{c}\text { Pre- } \\
\text { cisplatin } \\
\text { weight } \\
\text { loss } \\
(\%)\end{array}$ & $\begin{array}{c}\text { Day } 1 \\
\text { weight } \\
\text { loss } \\
(\%)\end{array}$ & $\begin{array}{c}\text { Day } 2 \\
\text { weight } \\
\text { loss } \\
(\%)\end{array}$ & $\begin{array}{c}\text { Day } 3 \\
\text { weight } \\
\text { loss } \\
(\%)\end{array}$ & $\begin{array}{c}\text { Serum } \\
\text { creatinine } \\
\text { (mg/dl) }\end{array}$ & $\begin{array}{c}\text { Blood } \\
\text { urea } \\
\text { nitrogen } \\
\text { (mg/dl) }\end{array}$ \\
\hline $\begin{array}{l}\text { Cis + High } \\
\text { Dose Eda }\end{array}$ & 86 & 29 & 25 & 25 & 23 & 22 & 13.79 & 13.79 & 20.69 & 24.14 & 0.39 & 94 \\
\hline $\begin{array}{l}\text { Cis + High } \\
\text { Dose Eda }\end{array}$ & 86 & 27 & 23 & 22 & 22 & 20 & 14.81 & 18.52 & 18.52 & 25.93 & 0.56 & 126 \\
\hline $\begin{array}{l}\text { Cis + } \\
\text { Matched } \\
\text { Dose Eda }\end{array}$ & 85 & 29 & 26 & 25 & DEAD & DEAD & 10.34 & 13.79 & DEAD & DEAD & NA & NA \\
\hline $\begin{array}{l}\text { Cis + } \\
\text { Matched } \\
\text { Dose Eda }\end{array}$ & 86 & 25 & 21 & 22 & 20 & 20 & 16.00 & 12.00 & 20.00 & 20.00 & 0.44 & 167 \\
\hline $\begin{array}{l}\text { Cis + } \\
\text { Matched } \\
\text { Dose Eda }\end{array}$ & 86 & 30 & 26 & 25 & 23 & 24 & 13.33 & 16.67 & 23.33 & 20.00 & 1 & 250 \\
\hline $\begin{array}{l}\text { Cis + } \\
\text { Matched } \\
\text { Dose Eda }\end{array}$ & 86 & 31 & 26 & 30 & 30 & 30 & 16.13 & 3.23 & 3.23 & 3.23 & 0.25 & 33 \\
\hline $\begin{array}{l}\text { Cis + } \\
\text { Matched } \\
\text { Dose Eda }\end{array}$ & 86 & 30 & 26 & 26 & 23 & 23 & 13.33 & 13.33 & 23.33 & 23.33 & 3.41 & 328 \\
\hline $\begin{array}{l}\text { Cis + } \\
\text { Matched } \\
\text { Dose Eda }\end{array}$ & 79 & 28 & 25 & 23 & 24 & 21 & 10.71 & 17.86 & 14.29 & 25.00 & 0.26 & 121 \\
\hline $\begin{array}{l}\text { Cis + } \\
\text { Matched } \\
\text { Dose Eda }\end{array}$ & 79 & 28 & 25 & 23 & 22 & 21 & 10.71 & 17.86 & 21.43 & 25.00 & 0.1 & 61 \\
\hline $\begin{array}{l}\text { Cis + } \\
\text { Matched } \\
\text { Dose Eda }\end{array}$ & 79 & 27 & 23 & 23 & 21 & 20 & 14.81 & 14.81 & 22.22 & 25.93 & 0.08 & 68 \\
\hline $\begin{array}{l}\text { Cis + } \\
\text { Matched } \\
\text { Dose Eda }\end{array}$ & 79 & 29 & 25 & 22 & 21 & 20 & 13.79 & 24.14 & 27.59 & 31.03 & 0.23 & 99 \\
\hline $\begin{array}{l}\text { Cis + } \\
\text { Matched } \\
\text { Dose Eda }\end{array}$ & 79 & 30 & 26 & 24 & 23 & 21 & 13.33 & 20.00 & 23.33 & 30.00 & 0.11 & 90 \\
\hline $\begin{array}{l}\text { Cis + Ctrl- } \\
\text { MNPs }\end{array}$ & 82 & 26 & 23 & 22 & 22 & 20 & 11.54 & 15.38 & 15.38 & 23.08 & 0.92 & 182 \\
\hline $\begin{array}{l}\text { Cis + Ctrl- } \\
\text { MNPs }\end{array}$ & 82 & 25 & 21 & 22 & 20 & 19 & 16.00 & 12.00 & 20.00 & 24.00 & 1.9 & 231 \\
\hline $\begin{array}{l}\text { Cis + Ctrl- } \\
\text { MNPs }\end{array}$ & 82 & 27 & 25 & 25 & 23 & 21 & 7.41 & 7.41 & 14.81 & 22.22 & 0.25 & 122 \\
\hline $\begin{array}{l}\text { Cis + Ctrl- } \\
\text { MNPs }\end{array}$ & 82 & 26 & 22 & 23 & 22 & 21 & 15.38 & 11.54 & 15.38 & 19.23 & 0.45 & 130 \\
\hline $\begin{array}{l}\text { Cis + Ctrl- } \\
\text { MNPs }\end{array}$ & 82 & 25 & 21 & 22 & 21 & 19 & 16.00 & 12.00 & 16.00 & 24.00 & 1.66 & 204 \\
\hline
\end{tabular}

C57BL/6 male 8-10 week old mice were injected intravenously with $50 \mathrm{mg} / \mathrm{kg}$ Eda-MNPs. Separately, four additional mice were injected intravenously with $0.2 \mathrm{mg} / \mathrm{kg}$ free edaravone. Each mouse was sacrificed $3 \mathrm{~h}$ after dosing; plasma and kidneys were collected for analysis.

For plasma preparation, $100 \mu \mathrm{l}$ of plasma from each mouse was protein-precipitated with $500 \mu \mathrm{l}$ cold acetonitrile before centrifugation at $4^{\circ} \mathrm{C}$ for $10 \mathrm{~min}$ at $12,700 \mathrm{RPM}$. The supernatant was dried and resuspended in $200 \mu \mathrm{l}$ acetonitrile for LC-MS analysis. For kidney tissue preparation, each kidney was placed into a homogenization tube with ceramic beads with $1.5 \mathrm{ml}$ acetonitrile. Tubes were run for $30 \mathrm{~s}$ at $6 \mathrm{~m} / \mathrm{s}$ on a BeadMill24 (Fisher). Tubes were cooled on ice for 5 min prior to centrifugation at 12,700 RPM for $15 \mathrm{~min}$ at $4^{\circ} \mathrm{C}$. The supernatant was dried and combined prior to completion. Following complete drying, $200 \mu \mathrm{l}$ of acetonitrile was added and vortex for $10 \mathrm{~min}$ prior to centrifugation under the same condition. The supernatant was carried on to LC-MS analysis.

Both sets of samples were run on a Shimadzu LCMS-8030 triple quadrupole LC-MS (Kyoto, Japan). Protocol validation determined edaravone MRM transitions: $175 \rightarrow 65.05$ (quant), $175 \rightarrow 44.05$, and $175 \rightarrow 77.05$. Solvent conditions were $0.1 \%$ formic acid in HPLC-grade water for the aqueous phase and $0.1 \%$ 


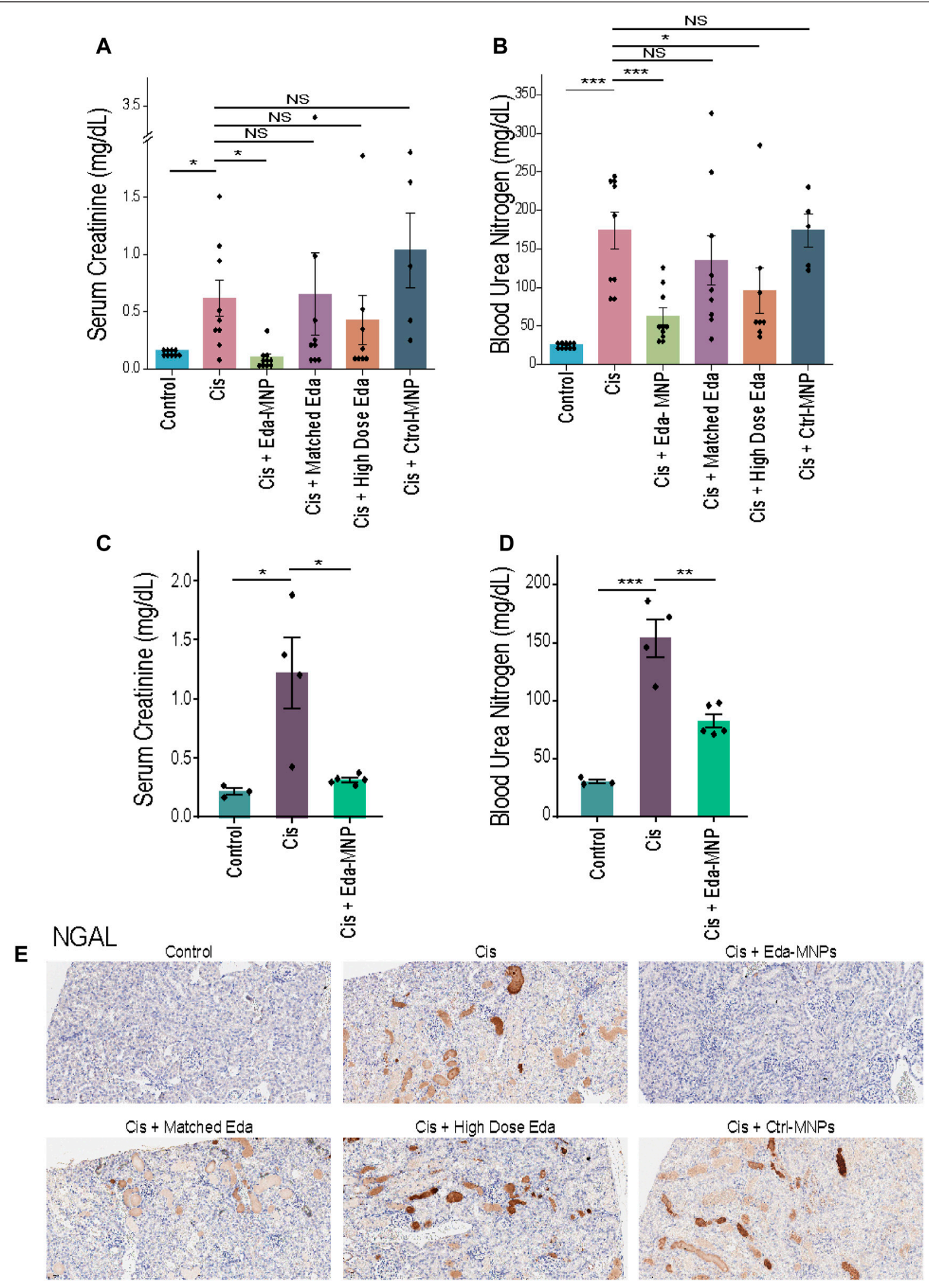

FIGURE 2 | Evaluation of renal function following treatment with Eda-MNPs and controls. (A) Serum creatinine of mouse groups induced to have Cl-AKI (Cis) and treated with Eda-MNPs or various controls. Bars represent the mean \pm SEM. One-way ANOVA with Sidak's posttest to compare to cisplatin control group. NS $=p>0.05 ;{ }^{*}=p<0.05$ (B) Blood urea nitrogen (BUN) of the same mouse groups as in panel (A). NS $=p>0.05 ;{ }^{*}=p<0.05 ;{ }^{* \star \star}=p<0.001$. (C) In non-fasted mice, serum creatinine of mouse groups with no AKI ( $N=3$ ), induced to have Cl-AKI (Cis) $(N=4)$, or treated with Eda-MNPs $(N=5)$. Bars represent the mean \pm SEM. Oneway ANOVA with Sidak's posttest to compare to cisplatin control group. Control vs Cis: $0.21 \pm 0.029$ vs $1.22 \pm 0.303 \mathrm{mg} / \mathrm{dll}, p={ }^{*}=0.013$; Cis vs Cis + Eda-MNP: (Continued) 
FIGURE 2 | $1.22 \pm 0.303$ vs $0.31 \pm 0.018 \mathrm{mg} / \mathrm{dl}, p={ }^{*}=0.012$ (D) In non-fasted mice, blood urea nitrogen (BUN) of the same mouse groups as in panel (C). One-way ANOVA with Sidak's posttest to compare to cisplatin control group. Control vs Cis: $30.33 \pm 1.86$ vs $154 \pm 16.27 \mathrm{mg} / \mathrm{dl}, p={ }^{\star \star \star}=0.000027$; Cis vs Cis + Eda-MNP: $154 \pm$ $16.27 \mathrm{vs} 82.6 \pm 5.91 \mathrm{mg} / \mathrm{dl}, p={ }^{* \star}=0.0074$. (E) $I \mathrm{HC}$ of NGAL expression in representative kidney sections from animals with each noted treatment, DAB detection (brown) and counterstained with haematoxylin (blue) ( $\times 10$ magnification).

A
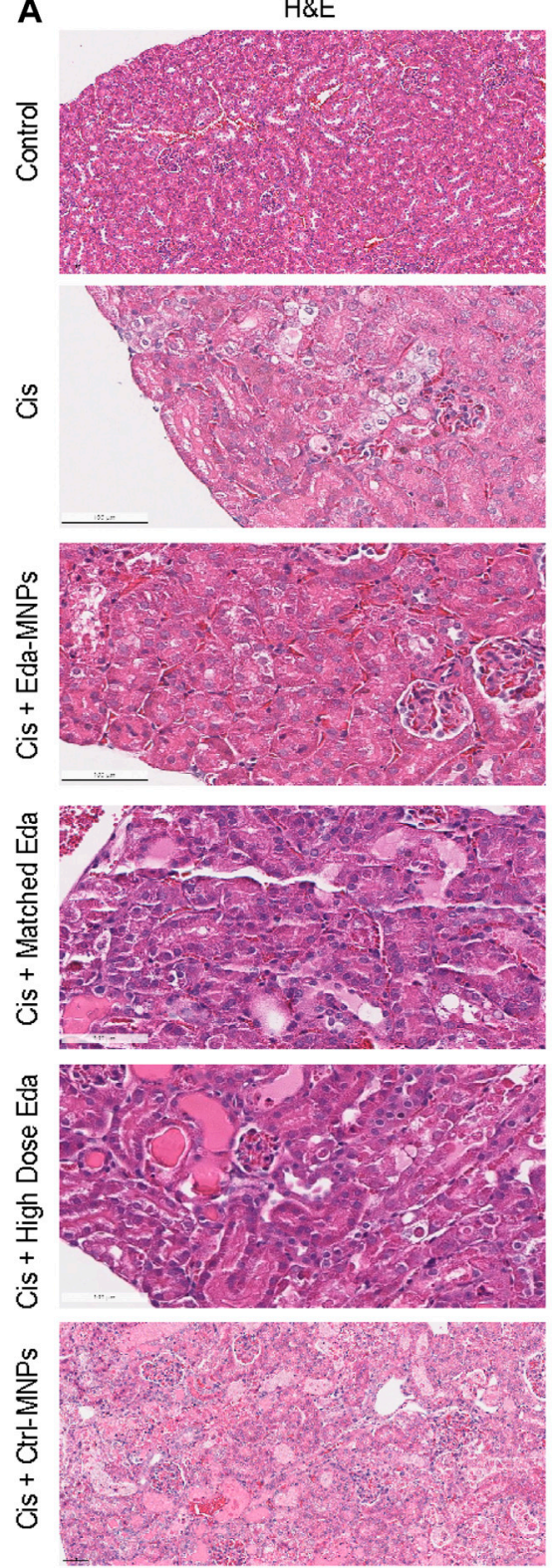

B
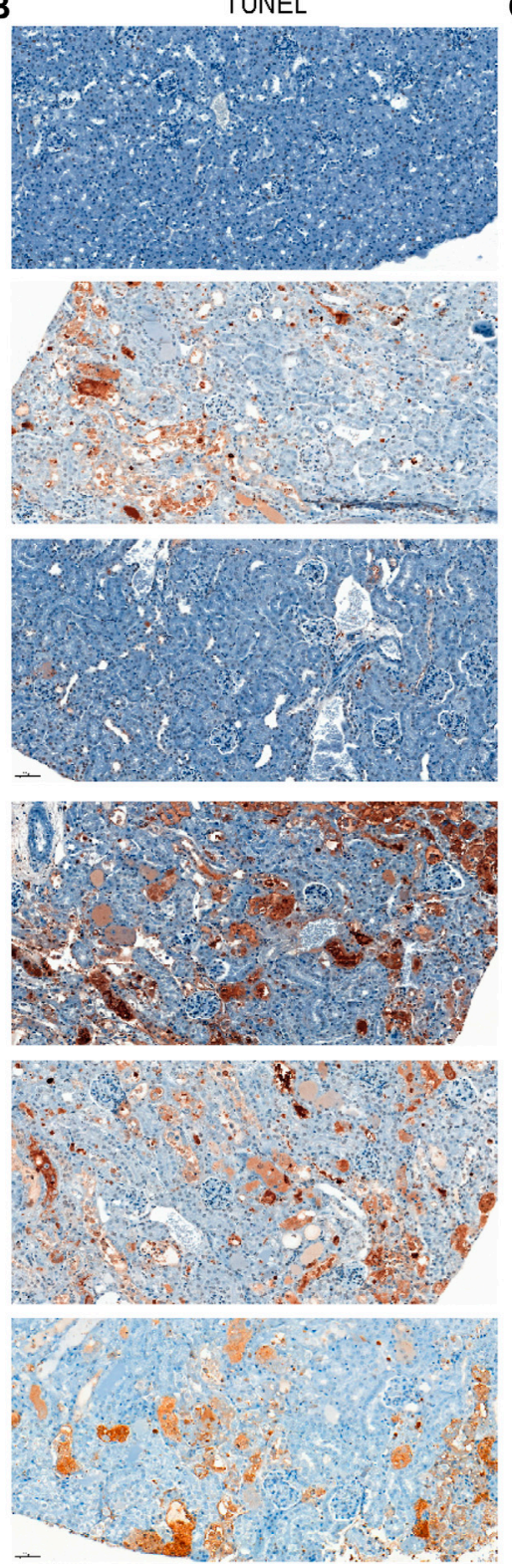

c
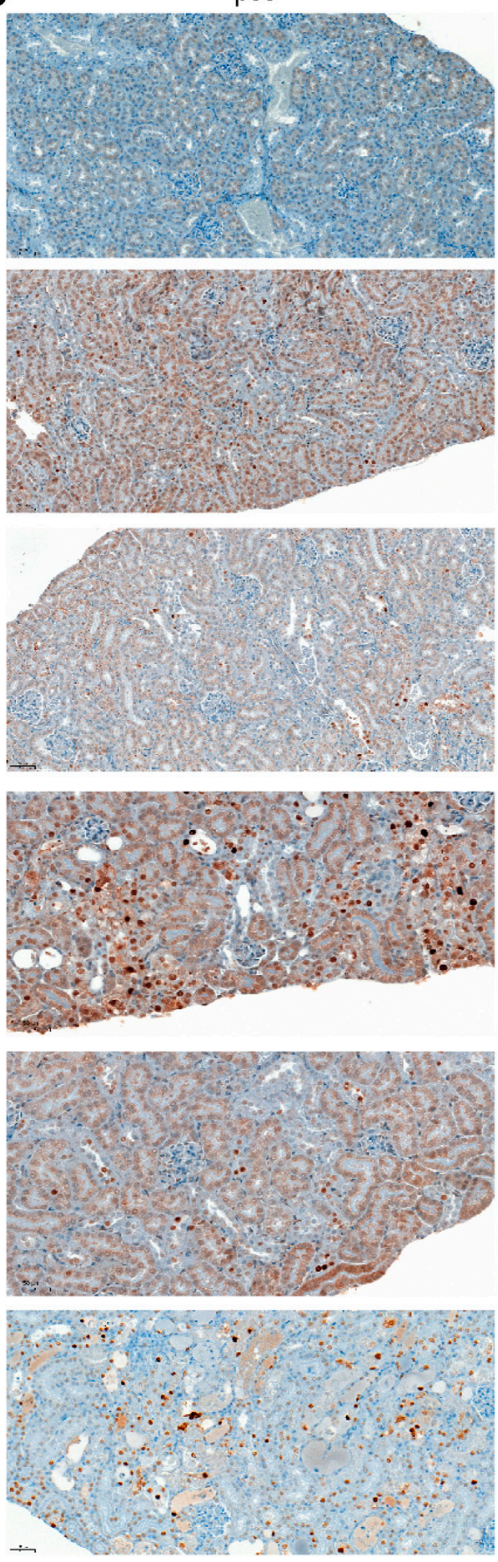

FIGURE 3 | Evaluation of cell injury and death following treatment. (A) Renal tissues were stained with haematoxylin (blue) and eosin (pink) (H\&E). (B) TUNEL detection in renal tissue via DAB (brown) and counterstained with haematoxylin (blue). (C) $I H C$ with DAB detection (brown) of an anti-p53 antibody and counterstained with haematoxylin (blue) in renal tissue (×10 magnification).

formic acid in methanol for the organic phase. Edaravone-spiked plasma calibrations were performed, with linear values ranging from $10 \mathrm{ng} / \mathrm{ml}$ to $10 \mu \mathrm{g} / \mathrm{ml}$. Limit of quantification values were
$10 \mathrm{ng} / \mathrm{ml}$ in plasma and approximately $7.6 \mathrm{pg} / \mathrm{mg}$ in kidney tissues, based on an average of kidney weights (approximately $261 \mathrm{mg}$ combined). 

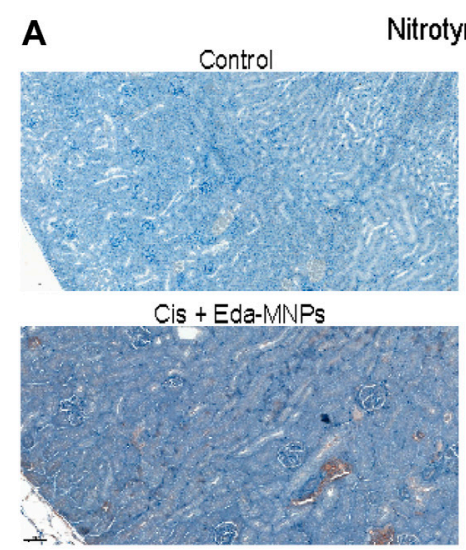

Cis + High Dose Eda

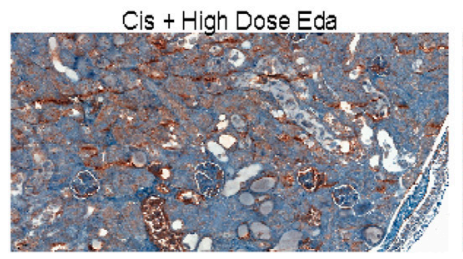

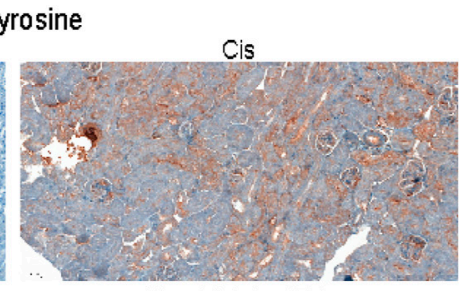

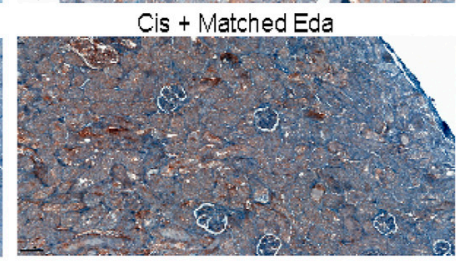

$\mathrm{Cis}+\mathrm{Ctrl}-\mathrm{MNN}$

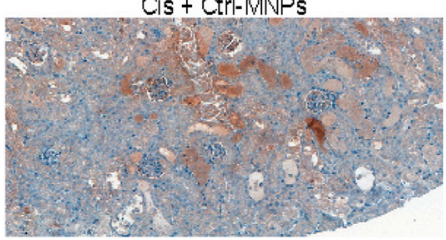

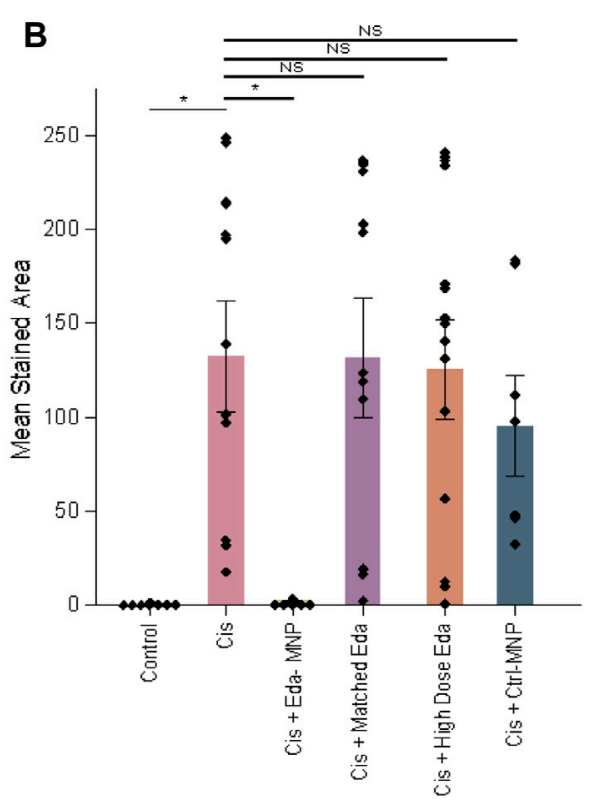

FIGURE 4 | Nitrotyrosine staining to evaluate pharmacodynamic outcomes of oxidative stress following Eda-MNP therapy. (A) IHC with DAB detection (brown) for 3-nitrotyrosine $\mathrm{IHC}$ and counterstained with haematoxylin (blue) (×10 magnification). (B) Semi-quantitative analysis of images from each treatment group. Bars represent mean \pm SEM. NS $=p>0.05 ;{ }^{*}=p<0.05$. Control vs Cis: $0.502 \pm 0.203$ vs $132.51 \pm 29.61, p={ }^{*}=0.013$. Cis vs Cis + Eda MNP: $132.51 \pm 29.61$ vs $2.094 \pm 0.594, p={ }^{*}=$ 0.036 .

\section{Renal Function Analysis}

At the time of animal sacrifice, blood was collected in serum preparation tubes (Becton Dickinson, Franklin Lake, NJ) and processed per manufacturer's recommendation. Serum was analyzed for urea nitrogen and creatinine levels via colorimetric methods in the MSKCC Laboratory for Comparative Pathology. For CI-AKI therapeutic statistical analysis, a one-way ANOVA with Sidak's posttest to compare to the cisplatin group was performed.

\section{Renal Histological Analysis}

Kidneys were formalin-fixed and prepared for histological analysis. Tissues were dehydrated and embedded in paraffin prior to obtaining $5 \mu \mathrm{m}$ sections, placing on a glass slide, and deparaffinizing. Sections were heat-retrieved at $\mathrm{pH} 6$ for $20 \mathrm{~min}$ and peroxide-blocked for $10 \mathrm{~min}$. Slides were incubated with each antibody or reagent below (1:400; Abcam, Cambridge, United Kingdom) for $50 \mathrm{~min}$ and then incubated with a biotinylated secondary antibody for $8 \mathrm{~min}$ (Vector Laboratories, Burlingame, CA). Slides were incubated with an anti-rabbit-conjugated horseradish peroxidase (HRP; Leica, Wetzlar, Germany) for $8 \mathrm{~min}$ and colorized with 3,3'diaminobenzidine (DAB; Ventana Medical Systems, Tuscon, AZ). Haematoxylin (Ventana Medical Systems) was used to counterstain slides prior to Permount (Fisher Scientific, Waltham, MA) coverslipping. Digital slide images were obtained with a Leica AT2 slide scanner.

A portion of renal tissue from each group above was formalinfixed and paraffin embedded as described above for histological analysis. Five parallel sections were obtained for each animal and subjected to the following treatments. 1) Hematoxylin and eosin (H\&E) (MSKCC Molecular Cytology Core Facility or HistoWiz Inc.); 2) Polyethylene-glycol (PEG) immunohistochemistry (IHC) to confirm particle localization (HistoWiz Inc., Brooklyn, NY) 3) NGAL IHC (HistoWiz); 4) Nitrotyrosine IHC (MSKCC Core); 5) p53 IHC (MSKCC core); 6) Terminal deoxynucleotidyl transferase-dUTP nick end labeling (TUNEL) IHC (MSKCC Core).

We performed semi-quantitative image analysis of 3nitrotyrosine staining using ImageJ Fiji similarly to as previously described for at least five individuals per group (Schindelin et al., 2012; Crowe and Yue, 2019). Briefly, digital slide images were captured at $\times 5$ magnification to include both renal cortex and medulla then opened in Fiji. Each file underwent color deconvolution using the "H DAB" vector option. The DAB vector for each image was then set to the same minimum and maximum threshold values to remove noise and ensure consistency. Measurements of the mean grey value were obtained for each thresholded vector, which was then plotted for each group. Measurements were subjected to a one-way ANOVA with Sidak's posttest to compare each group to the positive cisplatin control group.

\section{RESULTS AND DISCUSSION}

\section{MNP Formulation and Characterization}

To specifically deliver the free radical scavenger edaravone to the site of CI-AKI, we formulated kidney-targeted mesoscale nanoparticles to encapsulate the drug via nanoprecipitation as 
previously described (Williams et al., 2015; Williams et al., 2018). We formulated edaravone with the block-copolymer poly(lacticco- glycolic) acid (PLGA) conjugated to acid-terminated polyethylene glycol (PEG), or PLGA-PEG, obtaining therapeutic MNPs (Eda-MNPs). We also produced control MNPs with no encapsulated molecules via essentially the same procedure, apart from a lack of co-precipitate small molecule (Ctrl-MNPs).

To characterize MNPs, we first performed hydrodynamic diameter and $\zeta$-potential assays (Table 1). Eda-MNPs exhibited an average diameter ( $\mathrm{z}$ average) of $374.0 \pm 12.2 \mathrm{~nm}$ with a polydispersity index (PDI) of $0.337 .85 \%$ of these particles were within the $164-531 \mathrm{~nm}$ size range (Table 1; Figure 1A). Eda-MNPs exhibited a $\zeta$-potential of $-22.8 \pm 0.55 \mathrm{mV}$ in distilled water and $-18.2 \pm 0.51 \mathrm{mV}$ after incubation with $100 \%$ serum (Figure 1B). Ctrl-MNPs exhibited an average size of $332.0 \pm 7.56 \mathrm{~nm}$ with a PDI of $0.302 .74 \%$ of these particles fell within the 164-531 nm size range (Table 1; Figure 1A). CtrlMNPs exhibited a $\zeta$-potential in distilled water of $-24.7 \pm$ $0.61 \mathrm{mV}$ and $-13.2 \pm 0.7 \mathrm{mV}$ in $100 \%$ serum (Figure 1B). In two prior studies with MNPs, we found that particles of this composition with hydrodynamic diameters of 347.6 and 386.7 $\mathrm{nm}$, PDIs of approximately 0.3 , and surface charges in water of approximately $-20 \mathrm{mV}$ each had significant renal accumulation (Williams et al., 2015; Williams et al., 2018). We also found that the negative surface potential was partially abrogated after serum incubation (Williams et al., 2015; Williams et al., 2018). Therefore, we would expect that given the similar size and charge characteristics, as well as their interaction with serum, each of the formulations would exhibit similar renal targeting behavior.

We performed in vitro cargo release assays to evaluate the period over which MNPs release the encapsulated drug (Figure 1C). First, we found that Eda-MNPs contained 3.9 $\mu \mathrm{g}$ edaravone per $1 \mathrm{mg}$ of particle. Studies were performed in complete serum either at room temperature or $37^{\circ} \mathrm{C}$, with measurements taken frequently up to $6 \mathrm{~h}$ and daily to $72 \mathrm{~h}$. Eda-MNPs exhibited similar release characteristics at both room temperature and $37^{\circ} \mathrm{C}$ (Figure 1C). However, only about $30 \%$ of edaravone was released at room temperature up to $6 \mathrm{~h}$ and an additional 30\% was released between 24 and $48 \mathrm{~h}$. At elevated temperatures, the release rate was increased, though retaining the same pattern.

\section{Edaravone Pharmacokinetics Substantially Modulated by MNPs}

We first investigated the pharmacokinetics of MNPs loaded with the redox scavenger molecule edaravone (Eda-MNPs). We performed mass spectrometry studies on renal tissue and plasma in healthy mice treated with Eda-MNPs or dosematched free edaravone, sacrificed $3 \mathrm{~h}$ after injection. We found that measurable levels of edaravone $(7.6 \mathrm{ng} / \mathrm{ml}$ or above) were only present in the kidneys when encapsulated within MNPs (Figure 1D). Plasma levels of edaravone indicated the same trend (Figure 1E). These studies suggest that MNPs substantively modified the pharmacokinetics of edaravone, allowing substantial localization in the kidneys while also increasing the concentration available in circulation. Next, we performed immunohistochemistry (IHC) with an antiPEG antibody to stain for MNPs in the kidneys (Williams et al., 2015) (Figures 1F,G). In agreement with previous studies, we found that Eda-MNPs accumulate in primarily renal proximal tubular epithelial cells (Williams et al., 2015; Williams et al., 2018).

\section{Eda-MNPs Are Efficacious Against Cl-AKI}

To evaluate the potential clinical application of MNPs, we next sought to recapitulate a mouse model of CI-AKI. To do so, first we induced CI-AKI in $8-10$ weeks male C57BL/6 mice via intraperitoneal injection of $25 \mathrm{mg} / \mathrm{kg}$ cisplatin following $18 \mathrm{~h}$ of fasting/water deprivation.

To evaluate the therapeutic efficacy of Eda-MNPs against CI$\mathrm{AKI}$, we injected the particles $24 \mathrm{~h}$ following cisplatin injection and injury initiation (Table 2). We performed additional control experiments in which we injected Ctrl-MNPs (empty) and two concentrations of free edaravone: $0.2 \mathrm{mg} / \mathrm{kg}$ to match that which was administered in MNPs and $30 \mathrm{mg} / \mathrm{kg}$ to match prior work with this drug in rat models of kidney injury (Iguchi et al., 2004). These were compared against cisplatin-only and healthy control groups. Mice tolerated Eda-MNP, Ctrl-MNP, and the dosematched edaravone IV injections well, however after a single high dose of free edaravone, mice exhibited acute belabored breathing and sluggishness from which they eventually recovered. At $48 \mathrm{~h}$ after nanoparticle injection, and $72 \mathrm{~h}$ after cisplatin administration, we sacrificed the animals and obtained serum and kidney tissue for analysis.

We found that mice with CI-AKI treated with Eda-MNPs had kidney function and injury biomarkers similar to those in healthy control mice. We found that these mice had significantly lower serum creatinine (SCr) levels compared to untreated CI-AKI mice with levels at the baseline for healthy mice (Figure 2A; Table 2). We also found that the two free edaravone doses (dosematched and high dose) did not have a significant effect on $\mathrm{SCr}$ levels, nor did the Ctrl-MNPs (empty). We also evaluated renal health through blood urea nitrogen (BUN) levels, finding the same evidence of therapeutic efficacy from Eda-MNPs (Figure 2B; Table 2), with only the high dose of free edaravone showing a moderate statistically-significant benefit. It is clear from these serum-based markers of renal function that Eda-MNPs exhibit substantial benefit to renal function following cisplatin insult.

To further evaluate Eda-MNP efficacy, we performed a similar experiment in a different CI-AKI mouse model, in which the mice were not fasted or water deprived. Using the same Eda-MNP formulation, we again found a four-fold decrease in serum creatinine and two-fold decrease in BUN compared to mice receiving cisplatin only (Figures 2 C,D).

Finally, we evaluated expression of neutrophil gelatinaseassociated lipocalin (NGAL), a specific biomarker for acute kidney injury (Mishra et al., 2003; Supavekin et al., 2003). To do so, we performed IHC staining with an NGAL-specific antibody to determine expression in each group (Figure 2E). Using this metric, we found substantially less evidence of AKI in 
mice treated with Eda-MNP compared to mice treated with cisplatin alone or nanoparticle-alone or free drug controls. These results concur with the creatinine and BUN findings, demonstrating almost baseline levels NGAL expression in treated animals.

\section{Histological Analyses of Tubular Injury and Cell Death}

To further investigate the mechanism of Eda-MNPs therapeutic benefit on CI-AKI animals, we evaluated tubular injury and cell death via histology. First, we analyzed hematoxylin and eosin (H\&E) stains of each tissue, finding substantially reduced acute tubular necrosis, improved renal tubular architecture, and overall reduced evidence of AKI in mice treated with Eda-MNPs (Figure 3A). Next, we performed TUNEL staining to identify DNA fragmentation and apoptosis hallmarks of AKI (Havasi and Borkan, 2011) (Figure 3B), and IHC for p53 to identify loci of DNA damage and repair, overexpressed in AKI (Supavekin et al., 2003; Zhang et al., 2014) (Figure 3C). These findings clearly demonstrate an absence of apoptosis and DNA damage in mice treated with Eda-MNPs. This evidence suggests that an absence of tubular cell death was the product of tubular-specific delivery of edaravone afforded by Eda-MNPs and contributed to improved renal function.

\section{Pharmacodynamic Analysis of Kidney-Targeted Free Radical Scavenger Therapy}

Finally, we sought to evaluate the hypothesis that a reduction of cisplatin-induced oxidative stress due to tubular-specific delivery of edaravone was the mechanism of therapeutic function. To do so, we evaluated the presence of 3nitrotyrosine, which is the end-product of cisplatin-induced peroxynitrite formation in $\mathrm{AKI}$ and has previously been used to evaluate therapeutic outcomes involving this free radical pathway (Fiaccadori et al., 2004; Pan et al., 2014; Morigi et al., 2015). Nitrotyrosine has also previously been used as a pharmacodynamic endpoint for edaravone treatment in ALS clinical trials, as well as other laboratory investigations (Zhang et al., 2005; Yoshino and Kimura, 2006; Xi et al., 2007; Bandookwala et al., 2019). In addition, nitrotyrosine is widely recognized to be the direct result of cisplatin-induced cell and tissue damage, including ototoxicity and neurotoxicity in addition to nephrotoxicity (Giridharan et al., 2012; Rathinam et al., 2015; Jamesdaniel et al., 2016). Nitrotyrosine is also used as a primary endpoint with elevated levels in clinical and laboratory studies of both AKI (Thuraisingham et al., 2000; Qian et al., 2013; Sureshbabu et al., 2018) and other renal diseases (Thuraisingham et al., 2000; Fiaccadori et al., 2004). We therefore performed IHC for nitrotyrosine to evaluate the pharmacodynamics of free radical scavenger therapy. Representative IHC images showed that mice with CI-AKI had high levels of nitrotyrosine staining, whereas normal healthy mice had little to no staining (Figure 4A). Similar to prior results on renal function and cell death, we found that CI-
AKI mice that received Eda-MNPs exhibited almost baseline levels of nitrotyrosine production. We confirmed these representative results by performing semi-quantitative analysis of nitrotyrosine staining (Figure 4B). This data suggests that the MNP-enapsulated free radical scavenger conferred the intended effect at the site of injury. In free edaravone controls, little ROS scavenger likely reached the site of injury.

\section{CONCLUSION}

In these studies, we determined that edaravone-loaded MNPs exhibit striking therapeutic efficacy in a mouse model of CIAKI. We determined that this response was due to a reduction of proximal tubular cell death and damage as a result of edaravoneinduced free radical scavenging, only imparted by kidneyselective delivery by MNPs. We propose that, by administering edaravone via MNPs and not systemically, we would avoid interference with cancer treatment, as reactive oxygen species scavengers would do in cisplatin-treated patients (Sugihara and Gemba, 1986). We cannot discount, however, the possibility that some nanoparticle accumulation in the tumors may occur via the enhanced permeability and retention effect (EPR) and cause a reduction in cisplatin antitumor efficacy (Matsumura and Maeda, 1986; Sugihara and Gemba, 1986; Bertrand et al., 2014; Wang, 2015). Thus, additional studies will be necessary to evaluate to what extent this may occur, if any, though EPR-dependent nanoparticle uptake typically relies on smaller-sized particles than those studied here (Golombek et al., 2018). Though we hope that by reducing renal complications, we also would allow the patient to stay on the front-line cisplatin therapy for longer periods of time at higher doses, extending the chemotherapy's therapeutic window and effectiveness (Go and Adjei, 1999). This advancement has clear utility in clinical cancer management and nephrology.

Edaravone (marketed under the name Radicut) was originally developed in the 1980 s as a treatment for stroke (Abe et al., 1988) and has been on the market in Japan since 2001 for this indication (Group, 2003). It has been approved in Japan for ALS treatment since 2015 and in the United States since 2017 (Lapchak, 2010; Voelker, 2017). There have been several additional studies to evaluate the therapeutic efficacy of edaravone in other diseases, including traumatic brain injury and optic nerve injury, among others (Akiyama et al., 2017; Bailly, 2019; Shakkour et al., 2021). One such investigation found a moderate reduction (approximately 50\% for creatinine and BUN) of AKI in a rat model of cisplatininduced AKI (Iguchi et al., 2004). This data indeed supports our current findings from the "High-Dose Eda" free drug group, which found approximately a 33\% reduction in creatinine and BUN (though only BUN was statistically significant). Minor variability may have arisen due to the species used (rat vs mouse in our studies) and the dose of cisplatin (5 vs $25 \mathrm{mg} / \mathrm{kg}$ in our studies), among other differences. However, we believe that the marked increase in efficacy afforded by MNP-based kidney- 
targeted delivery of edaravone marks a substantial step toward clinical utility of edaravone use in CI-AKI.

There have been few prior efforts to develop kidney-targeted drug carriers for renal disease, with even less focus on CI-AKI (Alidori et al., 2016; Jiang et al., 2018). These studies further validate the need for further validation of nanomedicine approaches in the treatment of kidney disease (Williams et al., 2016). We expect similar pre-clinical pharmacokinetic studies to further the possibility of MNP clinical translation.

We found that edaravone-loaded MNPs exhibited substantial therapeutic potential in a murine model of CI-AKI. These characteristics substantially increase the likelihood of translation to the clinic for the treatment or prevention of CIAKI in patients with cancer. While these studies focused on acute kidney injury, we anticipate future work will evaluate this potential therapeutic specifically in animal models of chronic kidney disease, including cisplatin-induced and other etiologies (Eddy et al., 2012; Landau et al., 2019). However, the striking efficacy in preventing AKI found here portends a likely prevention of cisplatin-mediated CKD development and progression (Belayev and Palevsky, 2014; Fiorentino et al., 2018). We expect this approach to ultimately improve the quality of those patients' lives due to increased renal function after cisplatin therapy as well as a reduction in economic toll. We also expect this to allow those patients to stay on cisplatin as a front-line therapy, allowing increased survival from the underlying cancer.

\section{DATA AVAILABILITY STATEMENT}

The original contributions presented in the study are included in the article, further inquiries can be directed to the corresponding authors.

\section{ETHICS STATEMENT}

The animal study was reviewed and approved by the MSKCC IACUC.

\section{REFERENCES}

Abe, K., Yuki, S., and Kogure, K. (1988). Strong Attenuation of Ischemic and Postischemic Brain Edema in Rats by a Novel Free Radical Scavenger. Stroke 19, 480-485. doi:10.1161/01.str.19.4.480

Aird, W. C. (2007). Phenotypic Heterogeneity of the Endothelium: II. Representative Vascular Beds. Circ. Res. 100, 174-190. doi:10.1161/01.RES.0000255690.03436.ae Akiyama, G., Azuchi, Y., Guo, X., Noro, T., Kimura, A., Harada, C., et al. (2017). Edaravone Prevents Retinal Degeneration in Adult Mice Following Optic Nerve Injury. Invest. Ophthalmol. Vis. Sci. 58, 4908-4914. doi:10.1167/iovs.17-22250

Alidori, S., Akhavein, N., Thorek, D. L., Behling, K., Romin, Y., Queen, D., et al. (2016). Targeted Fibrillar Nanocarbon RNAi Treatment of Acute Kidney Injury. Sci. Transl Med. 8, 331ra39. doi:10.1126/scitranslmed.aac9647

Al-Mamgani, A., de Ridder, M., Navran, A., Klop, W. M., de Boer, J. P., and Tesselaar, M. E. (2017). The Impact of Cumulative Dose of Cisplatin on Outcome of Patients with Head and Neck Squamous Cell Carcinoma. Eur. Arch. Otorhinolaryngol. 274, 3757-3765. doi:10.1007/s00405-017-4687-4

\section{AUTHOR CONTRIBUTIONS}

RW, VT, LG, ES, EJ, and DH conceived of the experiments and designed the studies. RW, JS, EM, HT, VT, JC, MD, and JK conducted experiments and analyzed data. RW, EJ, and DH drafted the manuscript. All authors participated in manuscript preparation and approved its final form.

\section{FUNDING}

This work was supported in part by the NIDDK (R01-DK114321, R01 DK113088 to LJG), NCI (R01-CA215719), NCI Cancer Center Support Grant (P30-CA008748), NIH New Innovator Award (DP2-HD075698), the American Cancer Society Research Scholar Grant (GC230452), the Pershing Square Sohn Cancer Research Alliance, the Expect Miracles Foundation-Financial Services Against Cancer, the Anna Fuller Fund, the Louis V. Gerstner Jr Young Investigator's Fund, the Frank A. Howard Scholars Program, Cycle for Survival, the Alan and Sandra Gerry Metastasis Research Initiative, Mr. William H. Goodwin and Mrs. Alice Goodwin and the Commonwealth Foundation for Cancer Research, the Experimental Therapeutics Center, the Imaging and Radiation Sciences Program, and the Center for Molecular Imaging and Nanotechnology of Memorial Sloan Kettering Cancer. The authors would also like to think $\mathrm{R}$. Sandoval and B. Molitoris of the Indiana University Center for Biological Microscopy, NIH O’Brien Kidney Center (P30DK079312), for intravital imaging experiments. Electron microscopy experiments were supported by a NIH Shared Instrumentation Grant (S10RR27699) for Shared Resources. JK was supported by NIH Award F31-CA213814. EM, HT, and JC were supported NIH Award R25CA020449. RW was supported by the Ovarian Cancer Research Fund (Ann Schreiber Mentored Investigator Award 370463) and the American Heart Association Postdoctoral Fellowship (17POST33650043), The City College of New York Grove School of Engineering, PSC-CUNY Award ENHC-51-75, and the Oak Ridge Associated Universities Ralph E. Powe Junior Faculty Enhancement Award.

Awdishu, L., and Joy, M. S. (2016). Role of Pharmacogenomics in Kidney Disease and Injury. Adv. Chronic Kidney Dis. 23, 106-119. doi:10.1053/ j.ackd.2016.01.018

Bailly, C. (2019). Potential Use of Edaravone to Reduce Specific Side Effects of Chemo-, Radio- and Immuno-Therapy of Cancers. Int. Immunopharmacol 77, 105967. doi:10.1016/j.intimp.2019.105967

Bandookwala, M., Sahu, A. K., Thakkar, D., Sharma, M., Khairnar, A., and Sengupta, P. (2019). Edaravone-Caffeine Combination for the Effective Management of Rotenone Induced Parkinson's Disease in Rats: An Evidence Based Affirmative from a Comparative Analysis of Behavior and Biomarker Expression. Neurosci. Lett. 711, 134438. doi:10.1016/j.neulet.2019.134438

Belani, C. P. (2004). Seminars in Oncology. Philadelphia, PA: Elsevier, 25-33. Available at https://pubmed.ncbi.nlm.nih.gov/15726531/.

Belayev, L. Y., and Palevsky, P. M. (2014). The Link between Acute Kidney Injury and Chronic Kidney Disease. Curr. Opin. Nephrol. Hypertens. 23, 149-154. doi:10.1097/01.mnh.0000441051.36783.f3

Bellomo, R., Kellum, J. A., and Ronco, C. (2012). Acute Kidney Injury. Lancet 380, 756-766. doi:10.1016/S0140-6736(11)61454-2 
Bertrand, N., Wu, J., Xu, X., Kamaly, N., and Farokhzad, O. C. (2014). Cancer Nanotechnology: The Impact of Passive and Active Targeting in the Era of Modern Cancer Biology. Adv. Drug Deliv. Rev. 66, 2-25. doi:10.1016/ j.addr.2013.11.009

Brenner, B. M., Troy, J. L., Daugharty, T. M., Ueki, I., Nicholas, D., and Wong, C. (1971). On the Mechanism of Inhibition in Fluid Reabsorption by the Renal Proximal Tubule of the Volume-Expanded Rat. J. Clin. Invest. 50, 1596-1602. doi:10.1172/JCI106647

Cheng, J., Teply, B. A., Sherifi, I., Sung, J., Luther, G., Gu, F. X., et al. (2007). Formulation of Functionalized PLGA-PEG Nanoparticles for In Vivo Targeted Drug Delivery. Biomaterials 28, 869-876. doi:10.1016/j.biomaterials.2006.09.047

Crowe, A. R., and Yue, W. (2019). Semi-Quantitative Determination of Protein Expression Using Immunohistochemistry Staining and Analysis: An Integrated Protocol. Bio Protoc. 9, e3465. doi:10.21769/BioProtoc.3465

Di Lorenzo, G., Porta, C., Bellmunt, J., Sternberg, C., Kirkali, Z., Staehler, M., et al. (2011). Toxicities of Targeted Therapy and Their Management in Kidney Cancer. Eur. Urol. 59, 526-540. doi:10.1016/j.eururo.2011.01.002

Eddy, A. A., López-Guisa, J. M., Okamura, D. M., and Yamaguchi, I. (2012). Investigating Mechanisms of Chronic Kidney Disease in Mouse Models. Pediatr. Nephrol. 27, 1233-1247. doi:10.1007/s00467-011-1938-2

Fiaccadori, E., Maggiore, U., Rotelli, C., Giacosa, R., Lombardi, M., Sagripanti, S., et al. (2004). Plasma and Urinary Free 3-Nitrotyrosine Following Cardiac Angiography Procedures with Non-Ionic Radiocontrast media. Nephrol. Dial. Transpl. 19, 865-869. doi:10.1093/ndt/gfh039

Fiorentino, M., Grandaliano, G., Gesualdo, L., and Castellano, G. (2018). Acute Kidney Injury-Basic Research and Clinical Practice, 193. Basel, Switzerland: Karger Publishers, 45-54.

Gewin, L., Vadivelu, S., Neelisetty, S., Srichai, M. B., Paueksakon, P., Pozzi, A., et al. (2012). Deleting the TGF- $\beta$ Receptor Attenuates Acute Proximal Tubule Injury. J. Am. Soc. Nephrol. 23, 2001-2011. doi:10.1681/ASN.2012020139

Giridharan, V. V., Thandavarayan, R. A., Bhilwade, H. N., Ko, K. M., Watanabe, K., and Konishi, T. (2012). Schisandrin B, Attenuates Cisplatin-Induced Oxidative Stress, Genotoxicity and Neurotoxicity through Modulating NF-Kb Pathway in Mice. Free Radic. Res. 46, 50-60. doi:10.3109/10715762.2011.638291

Go, R. S., and Adjei, A. A. (1999). Review of the Comparative Pharmacology and Clinical Activity of Cisplatin and Carboplatin. J. Clin. Oncol. 17, 409-422. doi:10.1200/JCO.1999.17.1.409

Goldberg, R., and Dennen, P. (2008). Long-Term Outcomes of Acute Kidney Injury. Adv. Chronic Kidney Dis. 15, 297-307. doi:10.1053/j.ackd.2008.04.009

Golombek, S. K., May, J.-N., Theek, B., Appold, L., Drude, N., Kiessling, F., et al. (2018). Tumor Targeting via EPR: Strategies to Enhance Patient Responses. Adv. Drug Deliver Rev. 130, 17-38. doi:10.1016/j.addr.2018.07.007

Greenberg, A., and Cheung, A. K. (2005). Primer on Kidney Diseases. Philadelphia, PA: Elsevier Health Sciences.

Group, E. A. I. S. (2003). Effect of a Novel Free Radical Scavenger, Edaravone (MCI-186), on Acute Brain Infarction. Randomized, Placebo-Controlled, Double-Blind Study at Multicenters. Cerebrovasc. Dis. 15, 222-229. doi:10.1159/000069318

Han, S. J., Williams, R. M., D'Agati, V., Jaimes, E. A., Heller, D. A., and Lee, H. T. (2020). Selective Nanoparticle-Mediated Targeting of Renal Tubular Toll-like Receptor 9 Attenuates Ischemic Acute Kidney Injury. Kidney Int. 98 (1), 76-87. doi:10.1016/j.kint.2020.01.036

Han, S. J., Williams, R. M., Kim, M., Heller, D. A., D’Agati, V., Schmidt-Supprian, M., et al. (2020). Renal Proximal Tubular NEMO Plays a Critical Role in Ischemic Acute Kidney Injury. JCI Insight 5, e139246. doi:10.1172/ jci.insight. 139246

Havasi, A., and Borkan, S. C. (2011). Apoptosis and Acute Kidney Injury. Kidney Int. 80, 29-40. doi:10.1038/ki.2011.120

Hoste, E. A., and Schurgers, M. (2008). Epidemiology of Acute Kidney Injury: How Big Is the Problem? Crit. Care Med. 36, S146-S151. doi:10.1097/ CCM.0b013e318168c590

Humphreys, B. D., Cantaluppi, V., Portilla, D., Singbartl, K., Yang, L., Rosner, M. H., et al. (2015). Targeting Endogenous Repair Pathways after AKI. J. Am. Soc. Nephrol. 27 (4), 990-998. doi:10.1681/asn.2015030286

Iguchi, T., Nishikawa, M., Chang, B., Muroya, O., sato, E. F., Nakatani, T., et al. (2004). Edaravone Inhibits Acute Renal Injury and Cyst Formation in Cisplatin-Treated Rat Kidney. Free Radic. Res. 38, 333-341. doi:10.1080/ 10715760310001646886
Jamesdaniel, S., Rathinam, R., and Neumann, W. L. (2016). Targeting Nitrative Stress for Attenuating Cisplatin-Induced Downregulation of Cochlear LIM Domain Only 4 and Ototoxicity. Redox Biol. 10, 257-265. doi:10.1016/ j.redox.2016.10.016

Jiang, D., Ge, Z., Im, H. J., England, C. G., Ni, D., Hou, J., et al. (2018). DNA Origami Nanostructures Can Exhibit Preferential Renal Uptake and Alleviate Acute Kidney Injury. Nat. Biomed. Eng. 2, 865-877. doi:10.1038/s41551-0180317-8

Jo, S. K., Rosner, M. H., and Okusa, M. D. (2007). Pharmacologic Treatment of Acute Kidney Injury: Why Drugs haven't Worked and what Is on the Horizon. Clin. J. Am. Soc. Nephrol. 2, 356-365. doi:10.2215/CJN.03280906

Kim, J., Zarjou, A., Traylor, A. M., Bolisetty, S., Jaimes, E. A., Hull, T. D., et al. (2012). In Vivo Regulation of the Heme Oxygenase-1 Gene in Humanized Transgenic Mice. Kidney Int. 82, 278-291. doi:10.1038/ki.2012.102

Landau, S. I., Guo, X., Velazquez, H., Torres, R., Olson, E., Garcia-Milian, R. et al. (2019). Regulated Necrosis and Failed Repair in Cisplatin-Induced Chronic Kidney Disease. Kidney Int. 95, 797-814. doi:10.1016/ j.kint.2018.11.042

Lapchak, P. A. (2010). A Critical Assessment of Edaravone Acute Ischemic Stroke Efficacy Trials: Is Edaravone an Effective Neuroprotective Therapy? Expert Opin. Pharmacother. 11, 1753-1763. doi:10.1517/14656566.2010.493558

Latcha, S., Jaimes, E. A., Patil, S., Glezerman, I. G., Mehta, S., and Flombaum, C. D. (2016). Long-Term Renal Outcomes after Cisplatin Treatment. Clin. J. Am. Soc. Nephrol. 11, 1173-1179. doi:10.2215/CJN.08070715

Matsumura, Y., and Maeda, H. (1986). A New Concept for Macromolecular Therapeutics in Cancer Chemotherapy: Mechanism of Tumoritropic Accumulation of Proteins and the Antitumor Agent Smancs. Cancer Res. 46, 6387-6392.

Mishra, J., Ma, Q., Prada, A., Mitsnefes, M., Zahedi, K., Yang, J., et al. (2003). Identification of Neutrophil Gelatinase-Associated Lipocalin as a Novel Early Urinary Biomarker for Ischemic Renal Injury. J. Am. Soc. Nephrol. 14, 2534-2543. doi:10.1097/01.asn.0000088027.54400.c6

Morigi, M., Perico, L., Rota, C., Longaretti, L., Conti, S., Rottoli, D., et al. (2015). Sirtuin 3-Dependent Mitochondrial Dynamic Improvements Protect against Acute Kidney Injury. J. Clin. Invest. 125, 715-726. doi:10.1172/JCI77632

Müller, V., Losonczy, G., Heemann, U., Vannay, A., Fekete, A., Reusz, G., et al. (2002). Sexual Dimorphism in Renal Ischemia-Reperfusion Injury in Rats: Possible Role of Endothelin. Kidney Int. 62, 1364-1371. doi:10.1111/j.15231755.2002.kid590.x

Ozkok, A., and Edelstein, C. L. (2014). Pathophysiology of Cisplatin-Induced Acute Kidney Injury. Biomed. Res. Int. 2014, 967826. doi:10.1155/2014/967826

Pan, H., Shen, K., Wang, X., Meng, H., Wang, C., and Jin, B. (2014). Protective Effect of Metalloporphyrins against Cisplatin-Induced Kidney Injury in Mice. PLoS One 9, e86057. doi:10.1371/journal.pone.0086057

Qian, J., You, H., Zhu, Q., Ma, S., Zhou, Y., Zheng, Y., et al. (2013). Nitrotyrosine Level Was Associated with Mortality in Patients with Acute Kidney Injury. PLoS One 8, e79962. doi:10.1371/journal.pone.0079962

Rathinam, R., Ghosh, S., Neumann, W. L., and Jamesdaniel, S. (2015). CisplatinInduced Apoptosis in Auditory, Renal, and Neuronal Cells Is Associated with Nitration and Downregulation of LMO4. Cell Death Discov 1, 1-8. doi:10.1038/ cddiscovery.2015.52

Schindelin, J., Arganda-Carreras, I., Frise, E., Kaynig, V., Longair, M., Pietzsch, T., et al. (2012). Fiji: An Open-Source Platform for Biological-Image Analysis. Nat. Methods 9, 676-682. doi:10.1038/nmeth.2019

Shakkour, Z., Issa, H., Ismail, H., Ashekyan, O., Habashy, K. J., Nasrallah, L., et al. (2021). Drug Repurposing: Promises of Edaravone Target Drug in Traumatic Brain Injury. Curr. Med. Chem. 28, 2369-2391. doi:10.2174/ 0929867327666200812221022

Silver, S. A., and Chertow, G. M. (2017). The Economic Consequences of Acute Kidney Injury. Nephron 137, 297-301. doi:10.1159/000475607

Stamatiades, E. G., Tremblay, M. E., Bohm, M., Crozet, L., Bisht, K., Kao, D., et al. (2016). Immune Monitoring of Trans-Endothelial Transport by KidneyResident Macrophages. Cell 166, 991-1003. doi:10.1016/j.cell.2016.06.058

Sugihara, K., and Gemba, M. (1986). Modification of Cisplatin Toxicity by Antioxidants. Jpn. J. Pharmacol. 40, 353-355. doi:10.1254/jjp.40.353

Supavekin, S., Zhang, W., Kucherlapati, R., Kaskel, F. J., Moore, L. C., and Devarajan, P. (2003). Differential Gene Expression Following Early Renal Ischemia/reperfusion. Kidney Int. 63, 1714-1724. doi:10.1046/j.15231755.2003.00928.x 
Sureshbabu, A., Patino, E., Ma, K. C., Laursen, K., Finkelsztein, E. J., Akchurin, O., et al. (2018). RIPK3 Promotes Sepsis-Induced Acute Kidney Injury via Mitochondrial Dysfunction. JCI insight 3, e98411. doi:10.1172/jci.insight.98411

Thuraisingham, R. C., Nott, C. A., Dodd, S. M., and Yaqoob, M. M. (2000). Increased Nitrotyrosine Staining in Kidneys from Patients with Diabetic Nephropathy. Kidney Int. 57, 1968-1972. doi:10.1046/j.15231755.2000.00046.x

Voelker, R. (2017). Antioxidant Drug Approved for ALS. JAMA 317, 2363. doi:10.1001/jama.2017.6974

Walz, G., Budde, K., Mannaa, M., Nürnberger, J., Wanner, C., Sommerer, C., et al. (2010). Everolimus in Patients with Autosomal Dominant Polycystic Kidney Disease. N. Engl. J. Med. 363, 830-840. doi:10.1056/NEJMoa1003491

Wang, A. Z. (2015). EPR or No EPR? the Billion-Dollar Question. Sci. Translational Med. 7, 294ec112. doi:10.1126/scitranslmed.aac8108

Wei, Q., Wang, M. H., and Dong, Z. (2005). Differential Gender Differences in Ischemic and Nephrotoxic Acute Renal Failure. Am. J. Nephrol. 25, 491-499. doi:10.1159/000088171

Williams, R. M., Shah, J., Ng, B. D., Minton, D. R., Gudas, L. J., Park, C. Y., et al. (2015). Mesoscale Nanoparticles Selectively Target the Renal Proximal Tubule Epithelium. Nano Lett. 15, 2358-2364. doi:10.1021/nl504610d

Williams, R. M., Jaimes, E. A., and Heller, D. A. (2016). Nanomedicines for Kidney Diseases. Kidney Int. 90, 740-745. doi:10.1016/j.kint.2016.03.041

Williams, R. M., Shah, J., Tian, H. S., Chen, X., Geissmann, F., Jaimes, E. A., et al. (2018). Selective Nanoparticle Targeting of the Renal Tubules. Hypertension 71, 87-94. doi:10.1161/HYPERTENSIONAHA.117.09843

Xi, H., Akishita, M., Nagai, K., Yu, W., Hasegawa, H., Eto, M., et al. (2007). Potent Free Radical Scavenger, Edaravone, Suppresses Oxidative Stress-Induced Endothelial Damage and Early Atherosclerosis. Atherosclerosis 191, 281-289. doi:10.1016/j.atherosclerosis.2006.05.040

Xue, J. L., Daniels, F., Star, R. A., Kimmel, P. L., Eggers, P. W., Molitoris, B. A., et al. (2006). Incidence and Mortality of Acute Renal Failure in Medicare Beneficiaries, 1992 to 2001. J. Am. Soc. Nephrol. 17, 1135-1142. doi:10.1681/ ASN.2005060668

Yang, H. C., Zuo, Y., and Fogo, A. B. (2010). Models of Chronic Kidney Disease. Drug Discov. Today Dis. Models 7, 13-19. doi:10.1016/j.ddmod.2010.08.002

Yoshino, H., and Kimura, A. (2006). Investigation of the Therapeutic Effects of Edaravone, a Free Radical Scavenger, on Amyotrophic Lateral Sclerosis (Phase II Study). Amyotroph. Lateral Scler. 7, 241-245. doi:10.1080/ 17482960600881870
Zappitelli, M. (2008). Seminars in Nephrology. Philadelphia, PA: Elsevier, 436-446. Available at https://pubmed.ncbi.nlm.nih.gov/18790363/.

Zarjou, A., Sanders, P. W., Mehta, R. L., and Agarwal, A. (2012). Enabling Innovative Translational Research in Acute Kidney Injury. Clin. Transl Sci. 5, 93-101. doi:10.1111/j.1752-8062.2011.00302.x

Zhang, N., Komine-Kobayashi, M., Tanaka, R., Liu, M., Mizuno, Y., and Urabe, T. (2005). Edaravone Reduces Early Accumulation of Oxidative Products and Sequential Inflammatory Responses after Transient Focal Ischemia in Mice Brain. Stroke 36, 2220-2225. doi:10.1161/ 01.STR.0000182241.07096.06

Zhang, D., Liu, Y., Wei, Q., Huo, Y., Liu, K., Liu, F., et al. (2014). Tubular P53 Regulates Multiple Genes to Mediate AKI. J. Am. Soc. Nephrol. 25, 2278-2289. doi:10.1681/ASN.2013080902

Conflict of Interest: DH is a cofounder and officer with equity interest in Goldilocks Therapeutics, Inc., LipidSense, Inc., and Nirova BioSense, Inc. DH is a member of the scientific advisory boards of Concarlo Holdings, LLC, Nanorobotics, Inc., and Mediphage Bioceuticals, Inc. RW is a scientific advisor with equity interest in Goldilocks Therapeutics, Inc. EJ is a cofounder and chief medical officer with equity interest in Goldilocks Therapeutics Inc.

The remaining authors declare that the research was conducted in the absence of any commercial or financial relationships that could be construed as a potential conflict of interest.

Publisher's Note: All claims expressed in this article are solely those of the authors and do not necessarily represent those of their affiliated organizations, or those of the publisher, the editors and the reviewers. Any product that may be evaluated in this article, or claim that may be made by its manufacturer, is not guaranteed or endorsed by the publisher.

Copyright (C) 2022 Williams, Shah, Mercer, Tian, Thompson, Cheung, Dorso, Kubala, Gudas, de Stanchina, Jaimes and Heller. This is an open-access article distributed under the terms of the Creative Commons Attribution License (CC BY). The use, distribution or reproduction in other forums is permitted, provided the original author(s) and the copyright owner(s) are credited and that the original publication in this journal is cited, in accordance with accepted academic practice. No use, distribution or reproduction is permitted which does not comply with these terms. 